\title{
BIVARIATEALGEBRAIC INTEGERENCODED ARAI ALGORITHM FOR EXACT COMPUTATION OF DCT
}

\author{
Sumi Thomas ${ }^{1}$, M. Mathurakani ${ }^{2}$ \\ ${ }^{1}$ P.G Student, ${ }^{2}$ Formerly Scientist in DRDO, Professor, Electronics and Communication Engineering, Toc H Institute of \\ Science And Technology, Kerala, India
}

\begin{abstract}
Discrete Cosine Transform (DCT) function is widely used in many standard image and signal processing applications. Error free architectures with high throughput and reduced hardware area is a major concern.This paper surveys and particularly collates all the information available on arai algorithm that uses algebraic integer coded computation.Bivariate Algebraic Integer(AI) encoded 2-D $D C T$ algorithm ensuresquantization noise free implementation of -D DCT. This algorithm realizes an error-free 2-D DCT without using Final Reconstruction Steps(FRS) between row-column transforms, leading to an $8 \times 8$ 2-D DCT that is entirely free of quantization errors in AI basis,thereby avoiding the leakage of quantization noise between DCT channels.This architecture has infinite accuracy till the reconstruction step. The architecture enables low-noise high-dynamic range applications in image processing that requires full control of the finite-precision computation of the 2-D DCT. The $2 D$ DCT algorithm is simulated under fourdifferent conditions, on a gray coded 512 X512 image, and PSNR value is measured and compared using MATLAB.
\end{abstract}

Keywords: Algebraic Integer quantization, Discrete Cosine Transform (DCT), Scaled DCT/IDCT, Quantization Noise, Fixed point representation.

\section{INTRODUCTION}

High Dynamic Range image processing are under exponential growth and, therefore, the demand for applications capable of high dynamic range (HDR)imagingis accordingly increasing. Such HDR systems operating at high resolutions require an associate hardware capable of significant throughput at allowable area-power complexity. Efficient codec circuits capable of both high speeds of operation and high numerical accuracy are needed for next generation systems. Such systems may process massive amounts of input feeds, each at high resolution, with minimal noise and distortion while consuming as little energy as possible.

Straightforward techniques for spatial domain processingof images and videos are expensive and time consuming. An alternate approach is to process the image in compressed DCT domain, so that the output compressed stream corresponds to the output image and it confirms the syntax

of $8 \times 8$ block.

The major difficulties encountered are: the computational complexity of image compression and decompression algorithms, the high rate of data to be manipulated and increase in hardware area. For this reason there has been a great effort in recent years to develop fast algorithms that perform these tasks directly in the transform domain and thereby avoid the need of direct compression - the DCT which requires $38.7 \%$ of the execution time[14]. Usually, the
2-D DCT is computed by successive calls of the 1-D DCT applied to the columns of an $8 \times 8$ sub image, then to the rows of the transposed, resulting in intermediate calculations [18]. The very large-scale integration (VLSI) implementation of trigonometric transforms such as DCT and discrete Fourier transform is indeed an active research area [14][15].

An ideal eight-point 1-D DCT requires multiplications bynumbers in the form $\mathrm{c}[\mathrm{n}]=\cos (\mathrm{n} \pi / 16), \mathrm{n}=0,1 . \ldots 7$. Theseconstants impose computational difficulties in terms of number binary representation since they are not rational. For fast implementation we replace floating point numbers by appropriate dyadic rationalsby truncating or rounding offto approximate values. Both, rounding and approximation leads to truncation errors. Thus, instead of employing the exact value $c[n]$, a quantized value is considered. Clearly, this operation introduces errors.

In order to overcome this disadvantage Algebraic Integer Encoding scheme is adopted for DCT calculation[16]. With this scheme an error free representation of cosine functions become possible. Algebraic integers are roots of monicpolynomials that have integer coefficients with leading coefficient equal to unity. Here the cosine valuesarereplaced by a set of array of integers. After performing thecomputation in AI domain, a reconstruction algorithm is required to map the array of AI encoded functions back into their fixed point representation at a given precision [4]. 
In [11], AI-based procedures for the 2-D DCT are proposed. This architecture was based on the low-complexityArai algorithm [2], which formed the building-block of each1-D DCT using AI number representation. The Arai algorithm is a popular algorithm for video and image-processing applications because of its relatively low computational complexity. The eight-point Arai algorithm proposed in [10] needs only five multiplications to generate the eight output coefficients.

Thus, low complexity Arai algorithm is chosen as a foundation for proposing optimized architectures having lower complexity and lower noise. This paper extends the eightpoint 1-D AI-based DCT architecture into a fully parallel time-multiplexed 2-D architecture for $8 \times 8$ data blocks[4] which is further extended to $512 \times 512$ image and its inverse DCT calculation.

\section{PRELIMINARIES AND PAPER DESCRIPTION}

The 2D-DCT transformation a block $\{\mathrm{x}(\mathrm{m}, \mathrm{n})\}_{\mathrm{m}, \mathrm{n}=0}^{\mathrm{N}-1}$ in the spatial domain into a matrix of frequency components $\{\mathrm{X}(\mathrm{i}, \mathrm{j})$ \}$_{\mathrm{i}, \mathrm{j}=0}^{\mathrm{N}-1}$ according to the following equation:

$$
X(i, j)=\frac{1}{\sqrt{2 N}} C(i) C(j) \sum_{m=0}^{N-1 N-1} x(m, n) \cos \left(\frac{(2 m+1) i \pi}{2 N}\right) \cos \left(\frac{(2 n+1) j \pi}{2 N}\right)
$$

Where

$$
C(u)=\left\{\begin{array}{cc}
1 / \text { if } & u=0 \\
1 & \text { if } u \geq 1
\end{array}\right.
$$

And $\mathrm{N}$ is the length of the sequence.

Inverse DCT is calculated by:

$x(m, n)=\frac{1}{\sqrt{2 N}} \sum_{i=0}^{N-1} \sum_{i=0}^{N-1} c(i) c(j) x(i, j) \cos \left(\frac{(2 m+1) i \pi}{2 N}\right) \cos \left(\frac{(2 n+1) j \pi}{2 N}\right)$

DCT has the following properties:DCT has energy packing capability. DCT is orthogonal and separable, it leads to the reduction of spatial redundancy forthe input signal and has found wide applications in speech and image processing. The 2-Dimensional DCT, over a small block of pixels, has been widely used as afrequency analysis and compression algorithm in image processing standard likeMPEG-2.

\subsection{Theoretical Background}

The AI encoding for image processing was originally proposed by Cozzens and Finkelstein[5].They introduced a new approach for computing the DCT that uses the residue number system (RNS) in a ring of algebraic integers. In their work, the signal sample is represented by a set of typically four to eight small integers, combines, with the Residue Number System (RNS) to produce processors composed of simple parallel channels. This leads to a 1-D bivariate-encoded Arai DCT algorithm by Wahid and Dimitrov [11], [16]. Recently, subsequent contributions by Wahid using bivariateencoded 1-D Arai DCT blocks for row and column transforms of the 2-D DCT has led to practical area-efficient VLSI image-processing circuits with low-power consumption. This paper summarizes and compares the accuracy of both 1-D and 2-D DCT based on conventional fixed-point arithmetic as well as on AI encoding. The use of AI encoded array has the following advantages:

- The architecture is made error free by using exact integer representation instead of quantized value.

- Eliminate the need of intermediate reconstruction there by eliminate error propagation.

- Small array of integer values are used instead of truncated fixed point representation.

- Infinite precision and accuracy till the last reconstruction step.

- Saves hardware area by completely replacing multiplications by shift and negation operations thereby increasing the speed of computations.

- $\quad$ Reduces the total hardware area.

\subsection{AI Encoding And Decoding}

Algebraic integer encoded DCT is adopted to eliminate the quantization noise. Such a representation is based on a mapping function that links input numbers to integer arrays. AIs are root of monic polynomials with leading coefficient equal to unity [2]. The set of AIs are commutative, that is addition and multiplication operations are commutative and also satisfy distribution over addition. A general AI encoding mapping has the following format[4]:

$$
\mathbf{f}_{\text {enc }}(x ; z)=a=\left[\begin{array}{ll}
\mathbf{a}_{0} & \mathbf{a}_{1}
\end{array}\right]^{\mathrm{T}}
$$

Wherea is a multidimensional array of integers and $\mathbf{z}$ is afixed multidimensional array of AIs. It can be shown thatthere always exists an integer array such that any real number can berepresented with arbitrary precision [18]. For eg.,the irrational number $1-3 \sqrt{2}$ can be encoded as:

$$
f_{\text {enc }}\left(1-3 \sqrt{2} ;\left[\begin{array}{c}
1 \\
\sqrt{2}
\end{array}\right]\right)=\left[\begin{array}{c}
1 \\
-3
\end{array}\right]
$$

This forms an exact representation.Decoding operation is furnished by

$$
f_{\text {dec }}(\mathbf{a} ; \mathbf{z})=\mathbf{a} \cdot \mathbf{z}=\mathbf{a}_{0}+\mathbf{a}_{1} \cdot \mathbf{z}_{1}
$$

An exact AI array representation for all the DCT constants can be derived as shown in [11]. The Al representation has the following advantages: 
- Represents the required constants without error.

- Small integers are used andhence adopt a simple architecture and fast signal processing at an increased speed.

- Simple encoding and decoding operation.

\subsection{AI Encoded 1-D DCT}

Based on theseparability property of DCT and applying row column decomposition [7], the 2-D 8 x 8 DCT/IDCT can be realized by computing one-dimensional (1-D) 8 pointDCT/IDCT on all the rows followed by columns of thetotal $8 \times 8$ block. The computational complexity can be reduced by simplifying the DCT/IDCT coefficients by eliminating redundant coefficients. Thus for computation of DCT based on arai algorithm [4] we require the following coefficients:

$$
\begin{gathered}
C[4]=\quad \cos \left(\frac{4 \pi}{16}\right)= \\
C[6]=\cos \left(\frac{6 \pi}{16}\right)=\frac{\sqrt{2-}}{2} \\
C[2]-C[6]=\frac{\sqrt{2+\sqrt{2}}}{2}-\frac{\sqrt{2-}}{2} \\
C[2]+C[6]=\frac{\sqrt{2+\sqrt{2}}}{2}+\frac{\sqrt{2-}}{2}
\end{gathered}
$$

Byapplying 2D AI Encoding [4]

$$
\begin{array}{r}
\text { Let } z_{1}=\sqrt{2+\sqrt{2}}+\sqrt{2-} \\
\text { And } z_{2}=\sqrt{2+\sqrt{2}}-\sqrt{2-}
\end{array}
$$

The 2-D array for AI encoding is:

$$
z=\left(\begin{array}{cc}
1 & z_{1} \\
z_{2} & z_{1} z_{2}
\end{array}\right)
$$

This leads to a 2-D-encoded coefficient of the form

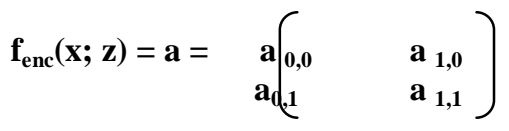

2D error free integer representation of the cosine values can be represented as shown in Table 1[4]. The employed integers are powers of two, and hence can be implemented by simple shift registers as shown in fig-1.For a given encoded number a, the decoding operation is expressed by:

$$
\mathbf{f}_{\text {dec }}(\mathbf{a} ; \mathbf{z})=\mathbf{a} \cdot \mathbf{z}=\mathbf{a}_{0,0}+\mathbf{a}_{1,0} \mathbf{z}_{1}+\mathbf{a}_{0,1} \mathbf{z}_{2}+\mathbf{a}_{1,1} \mathbf{z}_{1} \mathbf{z}_{2}
$$

Table -1: 2 D AI Encoded DCT constants

\begin{tabular}{|cc|cc|cc|cc|}
\hline \multicolumn{1}{|c|}{$\mathrm{C}[4]$} & \multicolumn{3}{|c|}{$\mathrm{C}[6]$} & \multicolumn{2}{c|}{$\mathrm{C}[2]-\mathrm{C}[6]$} & \multicolumn{2}{c|}{$\mathrm{C}[2]+\mathrm{C}[6]$} \\
\hline $\mathbf{0}$ & $\mathbf{0}$ & $\mathbf{0}$ & $\mathbf{1}$ & $\mathbf{0}$ & $\mathbf{0}$ & $\mathbf{0}$ & $\mathbf{2}$ \\
$\mathbf{0}$ & $\mathbf{1}$ & -1 & $\mathbf{0}$ & $\mathbf{2}$ & $\mathbf{0}$ & $\mathbf{0}$ & $\mathbf{0}$ \\
\hline
\end{tabular}

\section{AI ENCODED ARAI DCT ARCHITECTURE}

The image to be compressed is the input to the architecture in a row-wise order at the rate of one pixel ( 8 bits per pixel) per clock cycle as shown in the fig.1.The AI array of DCTcoefficients is multiplied with the input. Thus each input pixel $\mathbf{x}_{\mathbf{0}}$ after AI multiplication will be represented as an array as shown below:

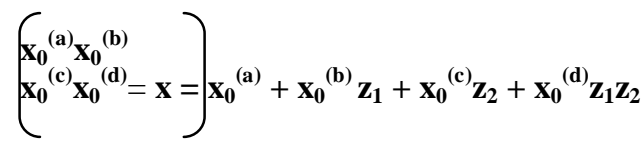

Where, (a),(b),(c) and (d) shows the position. Thus for each input pixel there will be 4 output channels, resulting in a total of 32 channels. Redundant bits can be eliminated, thereby resulting in only 22 channels as shown in the architecture in fig.1.

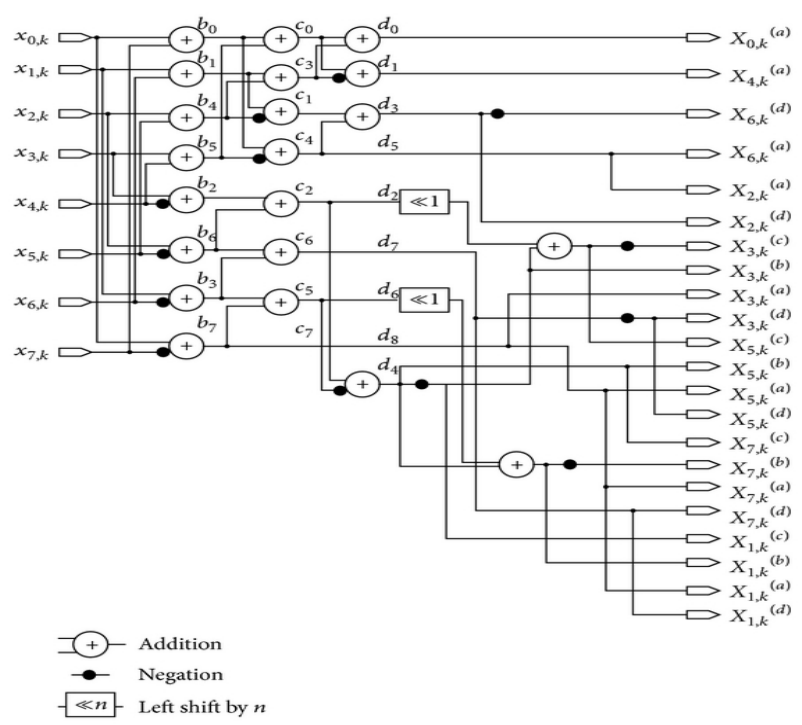

Fig -1: 1-D AI Arai DCT block [4]

Since the 2-D DCT is a separable transform, the row-column decomposition technique may be used to calculate the 2-D DCT. As a result, the functional units used for the first 1-D stage, can be re-used for the second 1-D DCT stage. In this case, the 2-D DCT of a $512 \times 512$ image hasbeen performed first by grouping it into blocks of $8 \times 8$ data. Then scaled arai 1-D DCT algorithm is applied to each row.This paper adopts the work of Agostini[11] that implemented Arai scaled 1-D 
DCT algorithm. The DCT coefficients produced by this algorithm are scaled values. Hence, we should multiply the scaled ones with a postscalar matrix to get the actual values [1].

\section{FINAL RECONSTRUCTION STEP}

The 22 channel representation corresponding to 8 pixel inputs must requires a reconstruction step to convert it back into corresponding 8 pixel DCT output. Intermediate reconstruction after each $1 \mathrm{D}$ DCT is eliminated inorder to prevent quantization noise cross coupling between DCT channels. The quantization noise is injected only at the final output. Therefore, noise signals are uncorrelated.

ArjunaMadanayake proposes two FRS based on1) optimized Dempster-Macleod multipliers, and 2) expansion

factor scaling[4]. DCT of an image is obtained in MATLAB using both the FRS methods and their PSNR values are shown in table 2 .

\subsection{Dempster- Macleod Method Based FRS}

In this method, the encoded elements aredecoded according to equation(15)as:

$X_{i, k}^{(q)}=X_{i, k}^{(q)(a)}+X_{i, k}^{(q)(b)} z_{1}+X_{i, k}^{(q)(c)} z_{2}+X_{i, k}^{(q)(d)} z_{1} z_{2}, q \square$ $\{a, b, c, d\}$

Values of $\mathrm{z}$ land $\mathrm{z}_{2}$ are given by equ.(10) and (11).The result is the $k^{\text {th }}$ row of the final DCT data Xi,k, i $=0,1, \ldots 7$.

For each value of $\boldsymbol{q}$, equation (16)can beunfolded as:

$$
\begin{aligned}
& X_{i, k}^{(a)}=X_{i, k}^{(a)(a)}+X_{i, k}^{(a)(b)} z_{1} \\
& +X_{i, k}^{(a)(c)} z_{2}+X_{i, k}^{(a)(d)} z_{1} z_{2} \\
& X_{i, k}{ }^{(b)} z_{1}=X_{i, k}{ }^{(b)(a)} z_{1}+X_{i, k}{ }^{(b)(b)} z_{1}{ }^{2} \\
& X_{i, k}{ }^{(c)} z_{2}=X_{i, k}{ }^{(c)(a)} z_{2}+X_{i, k}^{(c)(b)} z_{1} z_{2} \\
& +X_{i, k}^{(c)(c)} z_{2}^{2}+X_{i, k}^{(c)(d)} z_{1} z_{2}^{2} \\
& X_{i, k}{ }^{(d)} z_{1} z_{2}=X_{i, k}{ }^{(d)(a)} z_{1} z_{2}+X_{i, k}^{(d)(b)} z_{1}{ }^{2} z_{2} \\
& +X_{i, k}^{(d)(c)} z_{1} z_{2}^{2}+X_{i, k}^{(d)(d)} z_{1}^{2} z_{2}^{2}
\end{aligned}
$$

Thesum ofthe above given equ. returns $X_{i, k}$. The values $\mathrm{z}_{1}, \mathrm{z}_{2}$, $\mathrm{z}_{1} \mathrm{Z}_{2}$ used in the above given equations are irrational numbers. So the values recovered after this decoding are approximated fixed point values.

\subsection{FRS Based on Expansion Factor Scaling}

In this method, the quantities $\mathrm{z}_{1}, \mathrm{z}_{2}, \mathrm{z}_{1} \mathrm{z}_{2}$ are scaledinto integer values, thereby eliminating quantization error since we use integer values instead of fixed point values. This would facilitate the usage of simple integer arithmetic.
An expansion factor is defined as a real number $\alpha^{*}>1$ that satisfies the following minimization:

$$
\underset{\alpha>1}{\alpha^{*}=\operatorname{argmin}\|\alpha \cdot \zeta-\operatorname{round}(\alpha \cdot \zeta)\|}
$$

Where\|. \|is a given error measure and round (.) is the rounding function.In the range $\alpha \in[1,256]$,taking an optimal value $\alpha^{*}=167.2309$ [4] , we have the following scaling:

$$
\alpha^{*} *\left[\begin{array}{c}
z 1 \\
z 2 \\
z 1 z 2
\end{array}\right]=\left[\begin{array}{c}
437 \\
181 \\
473
\end{array}\right]
$$

However smaller values of $\alpha^{*}$ are more desirable because this requires much more simple hardware design. Now let us take $\alpha=4.5961[4]$,then:

$$
\alpha \cdot *\left[\begin{array}{c}
z 1 \\
z 2 \\
z 1 z 2
\end{array}\right]=\left[\begin{array}{c}
12 \\
5 \\
13
\end{array}\right]
$$

Considering a given expansion factor $\alpha$, the decoding operation given in equ. (16)can be generalized as:

$$
\begin{gathered}
X_{\mathrm{i}, \mathrm{k}}=1 / \alpha\left[\alpha \cdot X_{\mathrm{i}, \mathrm{k}}^{(\mathrm{a})}+\mathrm{n}_{1} \cdot X_{\mathrm{i}, \mathrm{k}}^{(\mathrm{b})}+\mathbf{n}_{2} \cdot \mathrm{X}_{\mathrm{i}, \mathrm{k}}^{(\mathrm{c})}\right. \\
\left.+\mathrm{n}_{3} \cdot \mathrm{X}_{\mathrm{i}, \mathrm{k}}^{(\mathrm{d})}\right]
\end{gathered}
$$

Where $\mathrm{n}_{1}, \mathrm{n}_{2}$, and $\mathrm{n}_{3}$ are the integer constants implied by the expansion factor $\alpha$. Here these constants are $\{437,181,473\}$, for $\alpha=\alpha^{*}$, and $\{12,5,13\}$, for $\alpha=\alpha$. The FRS is performed using both values of $\alpha$ and the PSNRvaluesare tabulated in Table -2.

\section{COMPARISON AND RESULTS}

We simulated the following four algorithms for DCT.

- Standard Arai Algorithm Based DCT

- $\quad$ AI Quantized DCT with $\alpha=4.5961$

- $\quad$ AI Quantized DCT with $\alpha=167.2309$

- Conventional Fixed Point Realization of DCT.

The image is decompressed back by applying conventional floating point IDCT in MATLAB.The simulation is done in MATLAB. The input is 512 x 512 lena.jpg image. The proposed algorithms have been applied to the input. The higher the value of PSNR, the better is the result. PSNR equal to infinity means the input image is perfectly reconstructed after IDCT. From Table-2 we conclude that, when we represent the DCT coefficients interms of there fixed point value and apply truncation with an accuracy of 8 bits, quantization noise is injected resulting in lossy compression and decompression. And hence the PSNR value is low. 
Table -2: Algorithms And PSNR values

\begin{tabular}{|c|c|}
\hline Algorithm & PSNR \\
\hline Arai DCT & INFINITY \\
\hline $\begin{array}{c}\text { Algebraic Integer DCT with } \\
\alpha=4.5961\end{array}$ & 41.3979 \\
\hline $\begin{array}{c}\text { Algebraic Integer DCT with } \\
\alpha=167.2309\end{array}$ & $\mathbf{5 2 . 7 5 3 2}$ \\
\hline Fixed Point DCT & $\mathbf{2 4 . 6 0 4 6}$ \\
\hline
\end{tabular}

Algebraic integer encoded DCT is adopted to eliminate the quantization noise. Such a representation is based on mapping function that links input numbers toan integer arrays instead of converting them into fixed point values.

Reconstruction step as discussed already is performed to map the DCT array to its corresponding fixed point representation. There is no intermediate reconstruction and hence prevents the leakage of quantization noise between DCT channels. From simulation results we can conclude that AIQ DCT has a better PSNR compared with fixed point realization. The proposed algorithms have been applied to the 512 × 512 image (fig. 2 :(a)) and the input image is reconstructed back successfully after performing both DCT (fig.2:(b)) and IDCT with floating point MATLAB (fig.2:(c)) .

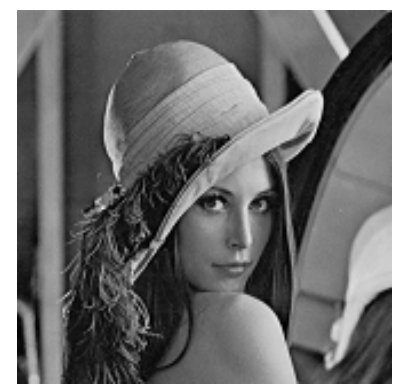

(a)

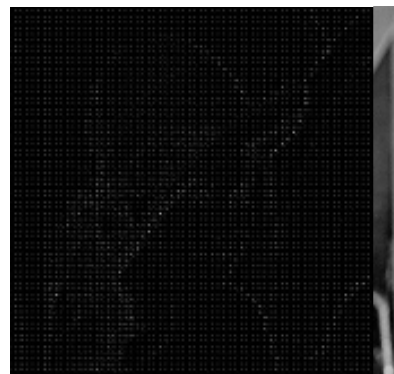

(b)

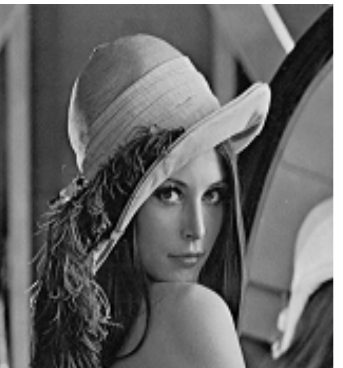

(c)
Fig -2: (a) INPUT IMAGE LENA 512 x 512 (b)DCT OUTPUT IMAGE (c) IDCT OUTPUT IMAGE

\section{CONCLUSIONS}

A time-multiplexed architecture proposed for the real-time computation of the bivariate AI encoded 2-D Arai DCT was simulated in MATLAB. The output of Arai AI DCT algorithm is obtained and the image is reconstructed back. The algorithm is not only completely multiplier-free but also quantization free up to the final output channels. The architecture does not have intermediate FRS sections between the column-wise and row-wise DCT operations. Thus the quantizationnoise appears only at the final output stage of the architecture. The location of the FRS at the final output stage resulted in the complete decoupling of quantization noise and provides the flexibility of user selectable accuracy. Simple shift and negation operations results in reduced hardware and ease of computation.

In this paper we simulated five algorithms and concluded that AI encoded DCT effectively reduces the overall arithmetic operations and allows multiplication-free,parallel and very fast implementation. With AI encoding DCT based image compression can be made error free.The use of integers in the encoding scheme also results exact reconstruction.

\section{REFERENCES}

[1] A.Pavani ,C.HemasundaraRao , A.Balaji Nehru , "Implementation of Pipelined Architecture Based on the DCT and Quantization For JPEG Image Compression" , International Journal Of Scientific Engineering \& Technology Research, Vol. 1, No.1,JulDec 2012.

[2] AmilaEdirisuriya , ArjunaMadanayake, Vassil S. Dimitrov, Renato J. Cintra 3 and JithraAdikari , "VLSI Architecture for 8-Point AI-based Arai DCT having LowArea-Time Complexity and Power at Improved Accuracy" , Journal of Low Power Electron. Appl. 2012.

[3] AntoninoTumeo, MatteoMonchiero, Gianluca Palermo, FabrizioFerrandi, Donatella Sciuto, "A Pipelined Fast 2D-DCT Accelerator for FPGA-based SoCs" IEEE Computer Society Annual Symposium on 2007.

[4] ArjunaMadanayake, Renato J. Cintra,,Denis Onen, Vassil S. Dimitrov, NilankaRajapaksha, L. T. Bruton, and AmilaEdirisuriya " A Row-Parallel 8×8 2-D DCT Architecture Using Algebraic Integer-Based Exact Computation", IEEE TRANSACTIONS ON CIRCUITS AND SYSTEMS FOR VIDEO TECHNOLOGY, VOL. 22, NO. 6, JUNE 2012.

[5] J. H. Cozzens and L. A. Finkelstein, "Range and error analysis fora fast Fourier transform computed over

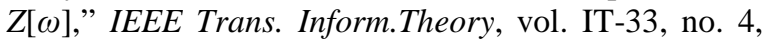
pp. 582-590, Jul. 1987.

[6] Jiun-In Guo, Rei-Chin Ju, and Jia-Wei Chen, "An Efficient 2-D DCT/IDCT Core Design UsingCyclic Convolution and Adder-Based Realization ", IEEE TRANSACTIONS ON CIRCUITS AND SYSTEMS 
FOR VIDEO TECHNOLOGY, VOL. 14, NO. 4, APRIL 2004.

[7] K. A. Wahid, M. Martuza, M. Das, and C. McCrosky, "Efficient hardware implementation of $8 \times 8$ integer cosine transforms for multiple video codecs," J. RealTime Image Process., vol. 6, no. 1, pp. 1-8, Jul. 2011.

[8] K. Wahid, "Error-free implementation of the discrete cosine transform," Ph.D. dissertation, Dept. Electric. Comput. Eng., Univ. Calgary, Calgary, AB, Canada, 2010.

[9] Khan A. Wahid, Vassil S. Dimitrov, and Graham A. Jullien, "On the Error-Free Realization of a Scaled DCT Algorithm and Its VLSI Implementation”, IEEE TRANSACTIONS ON CIRCUITS AND SYSTEMSII: EXPRESS BRIEFS, VOL. 54, NO. 8, AUGUST 2007.

[10] Khan Wahid, VassilDimitrov and Graham Jullien, "Error-Free Computation of 8x8 2-D DCT and IDCT using Two-Dimensional Algebraic Integer Quantization" , Proceedings of the 17th IEEE Symposium on Computer Arithmetic (ARITH'05) 2005 IEEE.

[11] L. Agostini, S. Bampi, "Pipelined Fast 2-D DCTArchitecture for JPEG Image Compression"Proceedings of the 14th Annual Symposium onIntegrated Circuits and Systems Design, Pirenopolis,Brazil. IEEE Computer Society 2001.

[12] Minyi Fu, V. S. Dimitrov, G. A. Jullien "An Efficient Technique for Error-Free Algebraic-Integer Encoding for High Performance Implementation of the DCT and IDCT",[2012].

[13] NeriMerhav and VasudevBhaskaran. "Fast Algorithms for DCT-Domain Down-Sampling and for Inverse Motion Compensation".

[14] Pramod Kumar Meher, Senior Member, IEEE, "Unified Systolic-Like Architecture for DCT and DST using Distributed Arithmetic" IEEE TRANSACTIONS ON CIRCUITS AND SYSTEMS-I: REGULAR PAPERS 12005.

[15] R. AsirSejoeNath\& D. Jessintha , "High Speed Systolic Array Discrete Cosine Transform Architecture With Fast Convolution", ISSN (Print) : 2319 - 2526, Volume-2, Issue-4, 2013.

[16] R. Baghaie and V. Dimitrov, "Systolic implementation of real-valueddiscrete transforms via algebraic integer quantization," Comput. Math.Applicat., vol. 41, no. 1, pp. 1403-1416, 2001.

[17] T. Suzuki and M. Ikehara, "Integer DCT based on direct-lifting of DCTIDCT for lossless-to-lossy image coding," IEEE Trans. Image Process., vol. 19, no. 11, pp. 2958-2965, Nov. 2010.

[18] V.S. Dimitrov, G.A. Jullien and W.C. Miller, "A New Dct Algorithm Based On Encoding Algebraic Integers".

\section{BIOGRAPHIES}

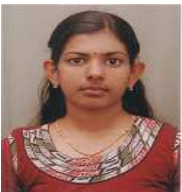

Sumi Thomas received the B.Tech degree in Electronics and Communication Engineering from Mar Baselios Christian College Of Engineering And Technology, Mahatma Gandhi University, Kerala, currently doing M.Tech in VLSI and Embedded Systems at Toc H Institute of Science and Technology, Cochin University.

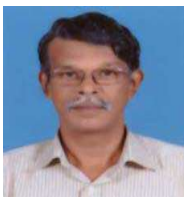

Prof. M. Mathurakanihas graduated from AlagappaChettiar College of Engineering and Technology of Madurai University and completed his masters from PSG college of Technology, Madras University. $\mathrm{He}$ has worked as a Scientist in Defence Research and Development Organization (DRDO) in the area of signal processing and embedded system design. He was honoured with the DRDO Scientist of the year award in 2003.Currently he is a professor in Toc H Institute of Science and Technology, Arakunnam. His area of research interest includes signal processing algorithms, embedded system implementations, reusable architecture and communication systems. 